\title{
Assessment of liver fibrosis by transient elastography and multi-parameters model in young children with chronic hepatitis B virus infection
}

Haiyan Luo ${ }^{1}$, Songxu Peng ${ }^{2}$, Wenxian Ouyang ${ }^{1}$, Yanfang Tan ${ }^{1}$, Tao Jiang ${ }^{1}$, Lian Tang ${ }^{1}$, Shuangjie Li ${ }^{*}$, Jun Qiu $^{3^{*}}$ and Changci Zhou ${ }^{4}$

\begin{abstract}
Objective: This study aimed to compare the diagnostic value of the single or combined applications of transient elastography (TE) and multivariate indicators with biopsy for the detection of liver fibrosis in children caused by chronic hepatitis B (CHB).

Methods: This study included 148 CHB children treated at Hunan Children's Hospital from January 1 st 2015 to December 31st 2018, aged from 0.83 to 14.58 years old. All patients underwent liver biopsy (LB), of which 43 patients underwent TE. Multiple clinical data, including aspartate aminotransferase (AST), alanine aminotransferase (ALT), Platelet (PLT), and HBV-deoxyribonucleic acid (HBV DNA) of all patients were collected. The diagnostic values for CHB of TE and its combinations with these indicators were measured. The patients were classified in two ways: no hepatic fibrosis group ( $F 0$ ) versus fibrosis group ( $F \geq 1)$, and no significant hepatic fibrosis group $(F<2)$ versus significant hepatic fibrosis group ( $\mathrm{F} \geq 2$ ). The statistical assessment was performed between groups within each classification to compare the diagnostic value of different parameters.
\end{abstract}

Results: The operating characteristic area under curve (AUC) of liver fibrosis diagnosed by liver stiffness measurement (LSM) which obtained by TE, AST-to-PLT ratio index (APRI), and fibrosis-4 index (FIB-4) were 0.740, 0.701, and 0.651, while the corresponding cut-off values were $5.9 \mathrm{kPa}, 0.50$, and 0.10 , respectively. The $A \cup C$ of significant liver fibrosis diagnosed by LSM, APRI and FIB-4 were $0.849,0.701$, and 0.509 , while the corresponding cut-off values were $8.4 \mathrm{kPa}$, 0.76 , and 0.08 , respectively. While with the combinations of LSM and APRI, LSM and FIB-4, LSM and APRI and FIB-4, APRI and FIB-4, the AUC of significant liver fibrosis were $0.866,0.855,0.869$, and 0.684 , respectively. The AUC of significant liver fibrosis diagnosed by the LSM was significantly higher than APRI and FIB-4.

Conclusions: The diagnostic value of transient elastography was better than that of APRI and FIB-4 for CHB children with significant liver fibrosis. In addition, TE also has relatively high application values on the diagnosis of patients with different degrees of liver fibrosis caused by CHB.

*Correspondence: lesjie@vip.sina.com; qiujuntrevor@163.com

${ }^{1}$ Department of Hepatology, Hunan Children's Hospital,

Changsha 410007, China

${ }^{3}$ Pediatrics Research Institute of Hunan Province, Hunan Children's

Hospital, Changsha 410007, China

Full list of author information is available at the end of the article

(C) The Author(s) 2022. Open Access This article is licensed under a Creative Commons Attribution 4.0 International License, which permits use, sharing, adaptation, distribution and reproduction in any medium or format, as long as you give appropriate credit to the original author(s) and the source, provide a link to the Creative Commons licence, and indicate if changes were made. The images or other third party material in this article are included in the article's Creative Commons licence, unless indicated otherwise in a credit line to the material. If material is not included in the article's Creative Commons licence and your intended use is not permitted by statutory regulation or exceeds the permitted use, you will need to obtain permission directly from the copyright holder. To view a copy of this licence, visit http://creativecommons.org/licenses/by/4.0/. The Creative Commons Public Domain Dedication waiver (http://creativeco mmons.org/publicdomain/zero/1.0/) applies to the data made available in this article, unless otherwise stated in a credit line to the data. 
Keywords: Chronic hepatitis B, Liver fibrosis, Diagnosis, Transient elastography, Multi-parameter indicators, Children

\section{Introduction}

Hepatitis B virus (HBV) infection is a widespread public health threat across the world. The hepatitis B serum epidemiological study held by the Chinese Center for Disease Control and Prevention (CDC) in 2014 discovered that the HBsAg detection rate was above $1.26 \%$ in children in China [1]. In addition, the age of the individual is the most important factor affecting the outcomes of HBV infection. The earlier the HBV infection occurs, the more likely to develop a chronic HBV infection. As mother-to-child transmission is the dominant way for HBV infection in China, accounting for $30-50 \%$ [1], mostly occurring in the perinatal period and transmitting through blood and body fluids of HBV positive mothers, it is necessary to pay more attention to children with chronic hepatitis B (CHB) [1]. CHB can further develop into liver fibrosis, a progressive disease state due to the excessive deposition of extracellular matrix in the liver. Since the failure of prompt treatment may lead to end-stage complications of liver disease such as decompensated liver cirrhosis, liver cancer, and liver failure [2], the control and removal of the etiology of liver fibrosis may be favorable for the disease reversion of liver fibrosis and even cirrhosis [3-5]. In clinical practices, the degree of liver fibrosis is of great significance to guide the treatment of $\mathrm{CHB}$, the monitoring of the disease, and the assessment of the final prognosis of the liver.

Currently, liver biopsy (LB) serves as the gold standard for the diagnosis and staging of liver fibrosis. However, LB is an invasive examination with complications such as right upper quadrant pain, bleeding, infection, pneumothorax, and so on [4]. Furthermore, the repeatability and compliance of operations are poor due to the sampling errors resulting from the limitation of samples, which greatly limits the clinical application, especially for children. The 2015 edition of the Chinese Guidelines for the Prevention and Treatment of Chronic Hepatitis B recommended a non-invasive diagnosis of liver fibrosis for the first time. The main diagnostic methods include fibrosis-4 index (FIB-4) and transient elastography (TE). However, the guideline did not propose non-invasive diagnostic indicators and techniques for liver fibrosis in children [1]. Although there are a lot of researches focused on non-invasive diagnosis models for liver fibrosis in CHB adults, little is about children. A recent study done by $\mathrm{Xu}$ et al. found that the liver stiffness measurement (LSM), the aspartate aminotransferase (AST)-platelet (PLT) ratio index (APRI), and FIB-4 were positively correlated with the fibrosis stage in $\mathrm{CHB}$ children aged 0-6 years old, and displayed the cut-off values of LSM for significant fibrosis and advanced fibrosis as $5.6 \mathrm{kPa}, 6.9 \mathrm{kPa}$, respectively [6], which was similar to the result of the study by $\mathrm{Xu}$ and his colleagues for children under 12 years old but it was different to the results for children above 12 years old [7]. Ulrike Teufel-Schäfer and his colleagues also found that TE showed a good correlation to the histological findings in children with hepatopathy [8]. Due to the small sample size, different operating instruments, limited data, and few studies on $\mathrm{CHB}$ in children, it is of great significance to conduct more studies to evaluate the non-invasive diagnostic index of liver fibrosis caused by CHB in children. Our study used samples from Hunan Children's Hospital to assess the diagnostic value of the single or combined applications of TE and multivariate indicators for liver fibrosis caused by CHB in children.

\section{Methods}

\section{Patient recruitment}

This study recruited 161 hospitalized patients diagnosed with CHB (aged 0-18 years old) at Hunan Children's Hospital from January 1st 2015 to December 31st 2018. Among them, 2 patients were excluded due to complicated infections with hepatitis $\mathrm{D}$ and 3 patients due to nonalcoholic fatty liver. At the same time, 3 patients who refused liver biopsy and 5 patients with incomplete data were also excluded. Finally, 148 eligible patients were enrolled (Fig. 1).They were divided into 5 stages F0, F1, F2, F3, and F4 according to the Scheuer system on pathological staging. After 2016,we introduced TE device and performed TE on 43 eligible patients within two weeks after the LB. They were also divided into 5 stages F0, F1, F2, F3, and F4 according to the Scheuer system on pathological staging.The serologic examinations were performed within two weeks after the LB. The sample size in our study was calculated by the formula as follow: $\mathrm{Nsp}=\left(\mathrm{Z}_{1-\alpha / 2} \times \sqrt{ } s e^{[1]} \times\right.$ $(1-s e) / 0.1)^{2}=(1.96 \times \sqrt{ } 0.915 \times(1-0.915) / 0.1)^{2} \approx 30$ [6].This study was approved by the Ethics Committee of Hunan Children's Hospital (IRB No. HCHLL-2019004), and obtained the formal written informed consent 


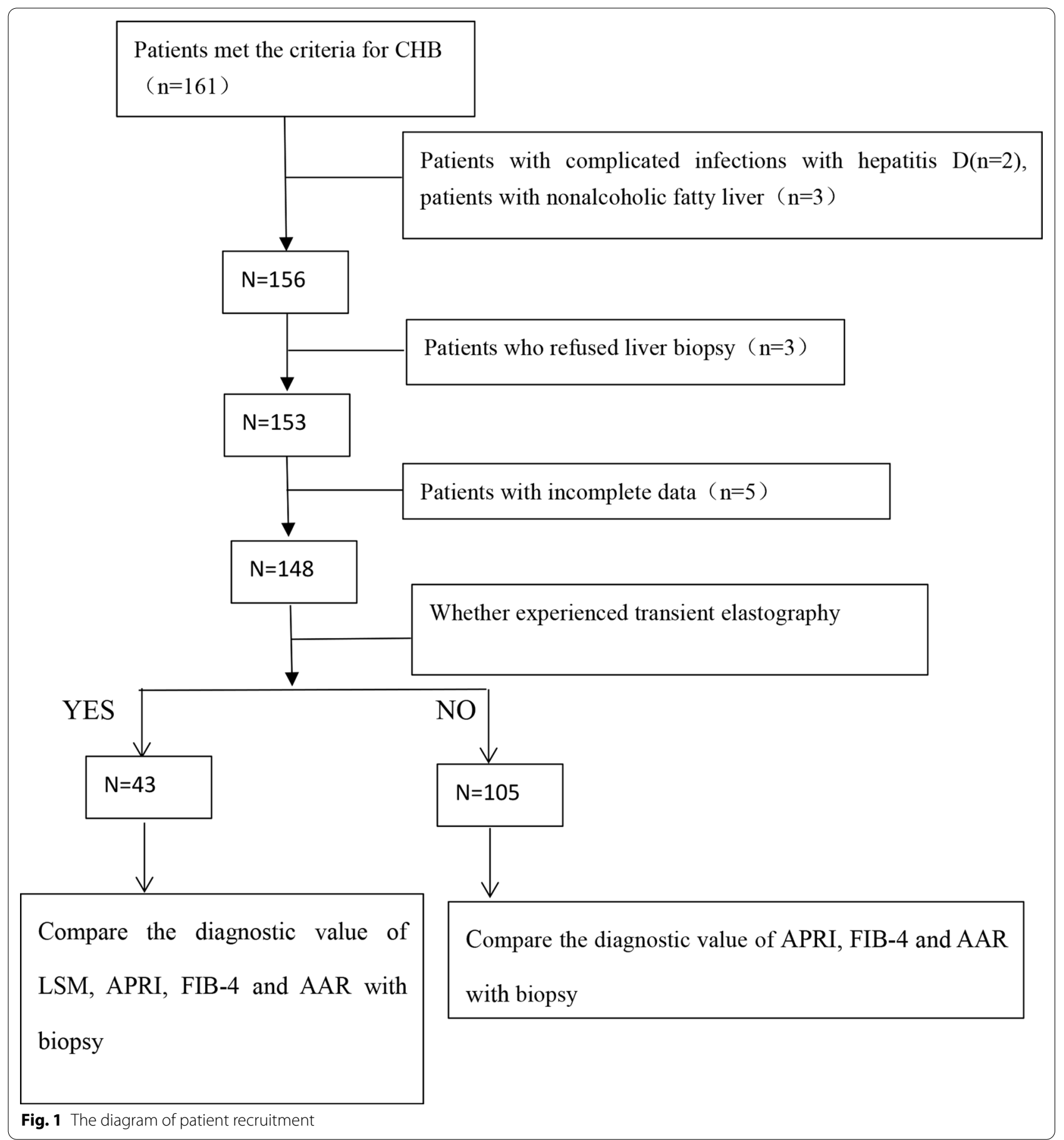

from all the parents and guardians of the participating children. For patients who experienced LB and who underwent both TE and LB, we classified them into two groups in the same ways: no hepatic fibrosis group (F0) and fibrosis group $(F \geq 1)$, no significant hepatic fibrosis group $(\mathrm{F}<2)$ and significant hepatic fibrosis group $(\mathrm{F} \geq 2)$.

\section{Detection of HBV-deoxyribonucleic acid (HBV DNA)}

Polymerase chain reaction (PCR) and fluorescence probe were used to detect the serum load of HBV DNA with the aid of ankle brachial index (ABI) 7500 instrument (made by Hunan Shengxiang Biological Science and Technology Ltd) and new HBsAg and anti-HBs kit. 
Detection of ALT and AST as well as peripheral platelets

Liver function was monitored by using the Bayer-2400 automatic biochemistry analyzer. The reference ranges of ALT and AST were from 0 to $40 \mathrm{U} / \mathrm{L}$. Peripheral platelets were assayed by the ADVIA2120 automated hematology analyzer and its corollary reagent manufactured by Bayer, Germany.

\section{Performance of liver biopsy (LB)}

The patients were maintained at the supine position or at the slightly lateral recumbent position to determine the puncture with B-ultrasound localization. Percutaneous liver biopsy was performed with Bard needle to obtain the liver tissue with $10 \%$ formaldehyde fixation fixed, HE stained and slices routinely prepared. After that, the same senior pathologist should read the slices in a unified manner and observe the pathological changes by the electron microscope to divide the degrees of liver fibrosis into stage F0 to F4 according to the Scheuer scoring system [1].

\section{Performance of liver stiffness measurement (LSM)}

The ultrasonic liver cirrhosis detector produced by Shenzhen Integrated Medical Technology Co, Ltd. was used, which is also a detection technique based on transient elastography. The intercostal space was chosen to measure the hardenability of liver parenchyma. The value of LSM was obtained after the measurement of effective values for ten times. The accuracy of the LSM value included shall be more than $60 \%$ with the deviation value being less than $1 / 3$ of the median. All procedures were performed by professionally trained physicians. We performed the examination of transient elastography when children fell asleep. For some children who cannot cooperate with the operation, we have sedated the children with the consent of their parents.

\section{Hepatic fibrosis scoring system: the Scheuer scoring systems}

F0: No fibrosis; F1: Fibrous expansion of portal areas; F2: Periportal fibrosis or portal-portal fibrous septa but intact architecture; F3: Fibrosis with architectural distortion but no obvious cirrhosis; F4: Probable or definite cirrhosis.

\section{The calculation formula for the non-invasive diagnostic model with multi-parameters}

$$
\begin{aligned}
\operatorname{APRI}= & \left(\operatorname{AST}(\mathrm{IU} / \mathrm{L}) / \text { upper level of normal value }(\mathrm{IU} / \mathrm{L}) / \operatorname{PLT}\left(10^{9} / \mathrm{L}\right)\right) \times 100 ; \\
& \text { FIB }-4 \text { Index }=(\text { age }(\text { year }) \times \operatorname{AST}(\mathrm{IU} / \mathrm{L})) /\left(\operatorname{PLT}\left(10^{9} / \mathrm{L}\right) \times \operatorname{ALT}^{1 / 2}(\mathrm{IU} / \mathrm{L}) ;\right. \\
& \text { AST }- \text { to }-\operatorname{ALT} \text { ratio }(\mathrm{AAR})=\operatorname{AST}(\mathrm{IU} / \mathrm{L}) / \operatorname{ALT}(\mathrm{IU} / \mathrm{L})
\end{aligned}
$$

\section{Statistical analysis}

SPSS 20.0 software and MedCalc software were used for statistical analysis. Median and inter-quartile range $(I Q R)$ were calculated for continued non-normal distribution measurement data as APRI, FIB-4 index, AAR, and LSM values. Categorical variables were expressed as the number and percentage of patients. Liver fibrosis stages F0 to F4 were identified by the gold standard for liver fibrosis according to LB.We classified patients into two groups in two ways: no hepatic fibrosis group (F0) and fibrosis group $(F \geq 1)$, no significant hepatic fibrosis group $(F<2)$ and significant hepatic fibrosis group $(F \geq 2)$. Based on the above described classification, the receiver operating characteristic (ROC) curves of the APRI, FIB-4 index, AAR, and LSM values were created and the area under the receiver operating characteristic curve $(A U C)$ was calculated within each group. Discrimination and calibration of the model were assessed by $A U C$, the predictive value was classified as low $(A U C=0.500-0.700)$, moderate $(A U C=0.700-0.900)$, or high $(A U C=0.900-1.000)$. Calibration across deciles of risk was evaluated using the Hosmer-Lemeshow goodness-offit test. For this test, a $P$-value $>0.05$ indicates good calibration [9]. Selecting the point corresponding to the maximum Yorden index was considered as the cut-off point. Then, using MedCalc software analyzed ROC curve to obtain the positive predictive value $(P P V)$, negative predictive value $(N P V)$, positive likelihood ratio $(+L R)$ and negative likelihood ratio $(-L R)$. The ROC contrast test was used to compare ROC curves. Excel software was used to calculate the $Z$ value based on the comparison of $A U C$ between different groups through the calculation formula $(Z=(S 1-S 2) /(S E 1$ $\left.\wedge 2+S E 2^{2}\right) \wedge 0.5$ ), where $S 1$ and $S 2$ indicate the area under ROC curve while $S E 1$ and $S E 2$ signify the corresponding standard error. Then the value of $P$ was calculated by the formula $P$ value $=1-$ NORMSDIST ( $Z$ value).

\section{Results}

Among $148 \mathrm{CHB}$ children who experienced LB, the youngest one was 0.83 years old and the oldest one was 14.58 years old as well as a median of 3.96 years old. In detail, $6(4.00 \%)$ were under 1 year old, $42(28.40 \%)$ were $1-3$ years old, $47(31.80 \%)$ were $3-5$ years old, $40(27.00 \%)$ were $5-10$ years old, and $13(8.80 \%)$ were above 10 years old.There were $28(18.92 \%)$ cases with F0, 94 (63.51\%) cases with F1, 19 (12.84\%) cases with F2, $5(3.38 \%)$ cases with F3 and 2 (1.35\%) cases with F4 (Table1). 
Table 1 Patient variables

\begin{tabular}{|c|c|c|c|}
\hline & Variable & Patients $^{a}(n=148)$ & Patients $^{\beta}(n=43)$ \\
\hline \multirow[t]{8}{*}{$\mathrm{F}=0$} & n (\%) & $28(18.92)$ & $9(20.93)$ \\
\hline & Male, n (\%) & $20(71.4)$ & $6(66.7)$ \\
\hline & HBeAg positive, $n(\%)$ & $28(100)$ & $9(100)$ \\
\hline & ALT (median, IQR, U/L) & $35.60(17.27,57.28)$ & $19.60(14.20,42.37)$ \\
\hline & AST (median, IQR, U/L) & $44.51(30.99,65.30)$ & $37.40(28.70,54.77)$ \\
\hline & PLT (median, $/ Q R, 10^{9} / \mathrm{L}$ ) & $305.50(257.75,337.00)$ & $310.00(245.00,345.50)$ \\
\hline & HBVDNA (median, IQR, IU/mL) & $3.05 \times 10^{7}\left(7.71 \times 10^{6}, 1.43 \times 10^{8}\right)$ & $3.11 \times 10^{7}\left(1.35 \times 10^{7}, 2.03 \times 10^{8}\right)$ \\
\hline & Age (median, $I Q R$, year) & $3.46(2.60,5.20)$ & $3.83(2.71,5.04)$ \\
\hline \multirow[t]{8}{*}{$F=1$} & $\mathrm{n}(\%)$ & $94(63.51)$ & $23(53.49)$ \\
\hline & Male, n (\%) & $67(71.3)$ & $16(69.6)$ \\
\hline & HBeAg positive, $n(\%)$ & $87(90.6)$ & $21(91.3)$ \\
\hline & ALT(median, IQR, U/L) & $57.45(30.93,96.53)$ & $60.10(20.00,89.90)$ \\
\hline & $\mathrm{AST}($ median, $/ \mathrm{QR}, \mathrm{U} / \mathrm{L}$ ) & $61.34(42.39,93.99)$ & $62.10(26.50,100.27)$ \\
\hline & PLT (median, IQR, 109/L) & $274.50(229.75,323.00)$ & $282.00(230.00,326.00)$ \\
\hline & HBVDNA (median, IQR, IU/mL) & $1.49 \times 10^{7}\left(2.56 \times 10^{6}, 4.01 \times 10^{7}\right)$ & $7.60 \times 10^{6}\left(4.56 \times 10^{6}, 3.00 \times 10^{7}\right)$ \\
\hline & Age (median, $I Q R$, year) & $4.40(2.98,6.58)$ & $5.83(3.83,9.00)$ \\
\hline \multirow[t]{8}{*}{$F=2$} & $n(\%)$ & $19(12.84)$ & $8(18.60)$ \\
\hline & Male, n (\%) & $16(84.2)$ & $6(75.0)$ \\
\hline & HBeAg positive, $n(\%)$ & $16(84.2)$ & $7(87.5)$ \\
\hline & ALT (median, IQR, U/L) & $79.00(52.60,117.40)$ & $75.40(45.81,106.87)$ \\
\hline & AST (median, IQR, U/L) & $78.19(43.10,203.90)$ & $77.95(47.77,106.87)$ \\
\hline & PLT (median, IQR, 109/L) & $261.00(226.00,287.00)$ & $272.50(248.00,414.50)$ \\
\hline & HBVDNA (median, IQR, IU/mL) & $7.28 \times 10^{6}\left(9.51 \times 10^{5}, 7.07 \times 10^{7}\right)$ & $7.28 \times 10^{6}\left(1.08 \times 10^{6}, 1.11 \times 10^{7}\right)$ \\
\hline & Age (median, $I Q R$, year) & $3.17(2.00,5.08)$ & $4.41(2.50,5.33)$ \\
\hline \multirow[t]{8}{*}{$F=3$} & $\mathrm{n}(\%)$ & $5(3.38)$ & $2(4.65)$ \\
\hline & Male, n (\%) & $3(60)$ & $2(100)$ \\
\hline & HBeAg positive, $n(\%)$ & $4(80)$ & $2(100)$ \\
\hline & ALT (median, IQR, U/L) & $95.20(60.65,182.55)$ & $79.50(95.20)$ \\
\hline & AST (median, IQR, U/L) & $105.30(73.70,290.95)$ & $383.80(105.30)$ \\
\hline & PLT (median, $/ Q R, 10^{9} / \mathrm{L}$ ) & $289.00(214.50,352.00)$ & $289.00(218.00)$ \\
\hline & HBVDNA (median, IQR, IU/mL) & $4.96 \times 10^{6}\left(4.29 \times 10^{5}, 5.86 \times 10^{7}\right)$ & $100.00\left(4.96 \times 10^{6}\right)$ \\
\hline & Age (median, IQR, year) & $3.67(2.75,8.16)$ & $4.08(3.25)$ \\
\hline \multirow[t]{8}{*}{$F=4$} & $\mathrm{n}(\%)$ & $2(1.35)$ & $1(2.33)$ \\
\hline & Male, n (\%) & $1(50)$ & $1(100)$ \\
\hline & HBeAg positive, $n(\%)$ & $1(50)$ & $0(0)$ \\
\hline & ALT (median, IQR, U/L) & $134.20(324.24)$ & 324.24 \\
\hline & AST (median, IQR, U/L) & $234.90(548.33)$ & 548.33 \\
\hline & PLT (median, IQR, 10/L) & $164(217)$ & 217 \\
\hline & HBVDNA (median, IQR, IU/mL) & $1.38 \times 105\left(8.43 \times 10^{6}\right)$ & $8.43 \times 10^{6}$ \\
\hline & Age (median, $I Q R$, year) & $1.42(1.17)$ & 1.17 \\
\hline
\end{tabular}

IQR inter-quartile range, $A L T$ alanine aminotransferase, $A S T$ aspartate aminotransferase, $A L P$ alkaline phosphatase, $P L T$ platelet, $H B e A g$ hepatitis $B$ e-antigen a:Patients underwent liver stiffness measurement (LSM) by TE and liver biopsy $\beta:$ Patients just underwent liver biopsy

Among the 43 patients who underwent both TE and $\mathrm{LB}$, the youngest was 1.08 years old, the oldest was 14.58 years old. 9 (21.00\%) were $1-3$ years old, 14 (32.50\%) were $3-5$ years old, 16 (37.20\%) were $5-10$ years old, and $4(9.30 \%)$ were above 10 years old. There were
9 (20.93\%) cases with F0, 23 (53.49\%) cases with F1, 8 (18.60\%) cases with F2, 2 (4.65\%) cases with F3 and 1 (2.33\%) case with F4 (Table1).

In terms of the disease course, the longest case reached 13 years while the shortest last for 1 week with a median 
value of 1.08 years. There were 107 males and 41 females, accounting for $72.30 \%$ and $27.70 \%$, respectively. The HBeAg positive cases were 136, making up for $91.89 \%$. Mothers of $130(87.84 \%)$ patients had a history of hepatitis $\mathrm{B}$ virus infection.The detailed information was shown in Table 1.

Based on LSM value, AUC for the diagnosis of liver fibrosis in CHB patients was 0.740 (95\% CI: 0.543$0.938)$, and the cut-off value, sensitivity $(\mathrm{Se})$, specificity $(S p)$, positive predictive value $(P P V)$, negative predictive value $(N P V)$, positive likelihood ratio $(+L R)$ and negative likelihood ratio $(-L R)$ for fibrosis were $5.9 \mathrm{kPa}, 94.12 \%, 55.56 \%, 88.90 \%, 71.40 \%, 2.12$ and 0.11 respectively. AUC for the diagnosis of liver fibrosis in CHB patients based on APRI value was 0.701 (95\% CI: $0.603-0.800)$, the cut-off value, $S e$, and $S p$ for fibrosis were $0.50,60.00 \%$, and $78.57 \%$, respectively. Based on the FIB-4 index, AUC for the diagnosis of liver fibrosis was 0.651 (95\% CI: $0.546-0.755)$, the cut-off value, $S e$, and $S p$ for fibrosis were $0.10,65.00 \%$, and $64.29 \%$, respectively. As for AAR value, $A U C$ for the diagnosis of liver fibrosis was 0.440 (95\% CI: $0.328-0.552$ ), the cut-off value, $S e$, and $S p$ for fibrosis were 0.90, 35.83\%, and $85.71 \%$, respectively. $A U C$ for the diagnosis of liver fibrosis in $\mathrm{CHB}$ patients based on a combination of LSM, APRI and FIB-4 was 0.771 (95\% CI: 0.580-0.942), which meant that the combination had a good discrimination of liver fibrosis, and a result of the HosmerLemeshow goodness-of-fit test for logistic regression confirmed that the combination was well calibrated $\left(\chi^{2}=0.170, P=0.264\right)$. The $A U C$ value $(0.740)$ of liver fibrosis diagnosed by LSM value was higher than that of APRI value (0.701), the differences of which showed no statistical significance $(Z$ was equal to 0.346 while $P$ was equal to 0.364$)$. The $A U C$ value (0.740) of liver fibrosis diagnosed by LSM value was higher than that of FIB-4 value (0.651), the differences of which showed no statistical significance $(Z$ was equal to 0.780 while
$P$ was equal to 0.218$)$. The $A U C$ value $(0.771)$ of liver fibrosis diagnosed by combining LSM and APRI as well as FIB-4 index was higher than that of LSM value (0.740), which showed no significant difference ( $Z$ was equal to 0.225 while $P$ was equal to 0.411$)$. The values of $S e, S p, P P V, N P V,+L R$ and $-L R$ in the diagnosis of liver fibrosis with biochemistry indicators were listed, all of which were shown in the following Table2, Figs. 2, and 3.

$A U C$ for the diagnosis of significant liver fibrosis in $\mathrm{CHB}$ patients based on LSM value was 0.849 (95\% CI: 0.713-0.986), and the cut-off value, $S e, S p, P P V$, $N P V,+L R$ and $-L R$ for significant liver fibrosis were $8.4 \mathrm{kPa}, 81.82 \%, 78.12 \%, 56.20 \%, 92.60 \%, 3.74$ and 0.23 respectively. $A U C$ for the diagnosis of significant liver fibrosis in CHB patients based on APRI value was 0.701 (95\% CI: $0.591-0.810)$, the cut-off value, $S e$, and $S p$ for fibrosis were $0.76,61.54 \%$, and $72.95 \%$, respectively. Based on the FIB-4 index, $A U C$ for the diagnosis of significant liver fibrosis was 0.509 (95\% CI: 0.388-0.630), and the cut-off value, $S e$, and $S p$ for fibrosis were 0.08 , $84.62 \%$, and $27.87 \%$, respectively. As for AAR value, $A U C$ for the diagnosis of significant liver fibrosis was 0.458 (95\% CI: 0.329-0.586), the cut-off value, $\mathrm{Se}$, and $S p$ for fibrosis were $1.13,69.23 \%$, and $48.36 \%$, respectively. $A U C$ for the diagnosis of significant liver fibrosis in CHB patients based on a combination of LSM, APRI and FIB-4 was 0.869 (95\% CI: 0.741-0.998), which means that the combination had a good discrimination of significant liver fibrosis, and a result of the Hosmer-Lemeshow goodness-of-fit test for logistic regression confirmed that the combination was well calibrated $\left(\chi^{2}=4.619\right.$, $P=0.797)$. The $A U C$ value (0.849) of significant liver fibrosis based on LSM value was higher than that of APRI value (0.701), the differences of which reached statistical significance ( $Z$ were equal to 1.650 while $P$ were equal to 0.049$)$. The $A U C$ value (0.849) of significant liver fibrosis based on LSM value was higher than that of

Table 2 The area under ROC curve and its relevant parameters of liver fibrosis diagnosed by four non-invasive diagnostic indicators single or in combination

\begin{tabular}{|c|c|c|c|c|c|c|c|c|c|c|c|c|c|}
\hline Parameters & $\mathbf{n}$ & $A U C$ & $P$ & $95 \% \mathrm{Cl}$ & Cut-off & $\mathrm{Se}(\%)$ & Sp (\%) & PPV (\%) & NPV (\%) & $+\mathrm{LR}$ & $-\mathrm{LR}$ & $P$ & $x^{2}$ \\
\hline APRI & 148 & 0.701 & 0.001 & $0.603-0.800$ & 0.50 & 60.00 & 78.57 & 92.30 & 31.40 & 2.80 & 0.51 & & \\
\hline AAR & 148 & 0.440 & 0.326 & $0.328-0.552$ & 0.90 & 35.83 & 85.71 & 91.50 & 23.80 & 2.51 & 0.75 & & \\
\hline FIB-4 & 148 & 0.651 & 0.013 & $0.546-0.755$ & 0.10 & 65.00 & 64.29 & 88.60 & 30.00 & 1.82 & 0.54 & & \\
\hline APRI + FIB-4 & 148 & 0.703 & 0.001 & $0.605-0.801$ & 0.80 & 51.67 & 89.29 & 95.40 & 30.10 & 4.82 & 0.54 & 0.560 & 6.788 \\
\hline LSM & 43 & 0.740 (1) & 0.028 & $0.543-0.938$ & $5.9 \mathrm{kPa}$ & 94.12 & 55.56 & 88.90 & 71.40 & 2.12 & 0.11 & & \\
\hline$A P R I+L S M$ & 43 & 0.761 & 0.017 & $0.580-0.942$ & 0.57 & 94.12 & 55.56 & 88.90 & 71.40 & 2.12 & 0.11 & 0.636 & 6.104 \\
\hline FIB-4 + LSM & 43 & 0.758 & 0.018 & $0.555-0.961$ & 0.58 & 94.12 & 55.56 & 88.90 & 71.40 & 2.12 & 0.11 & 0.384 & 8.529 \\
\hline $\mathrm{LSM}+\mathrm{APRI}+\mathrm{FIB}-4$ & 43 & 0.771 & 0.017 & $0.580-0.942$ & 0.53 & 97.06 & 55.56 & 89.20 & 83.30 & 2.18 & 0.05 & 0.264 & 0.170 \\
\hline
\end{tabular}

(1)Compared with APRI, $Z=0.346, P=0.364$ (1) compared with FIB-4, $Z=0.780, P=0.218$; (1) compared with LSM $+\mathrm{APRI}+\mathrm{FIB}-4, Z=0.225, P=0.411$ 


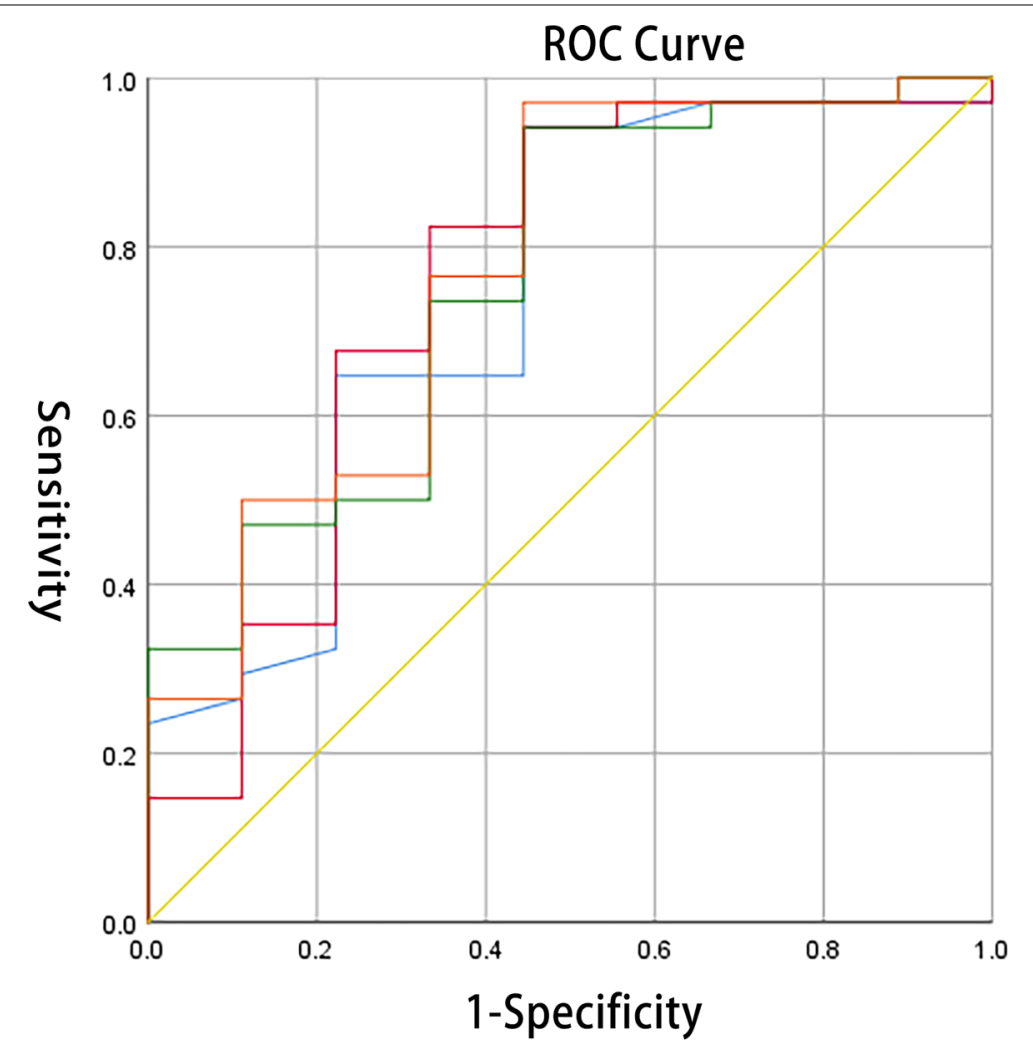

\section{Source of Curves}

- LSM

- FIB-4+LSM

APRI+LSM

APRI+FIB-4+LSM

Reference line

Diagonal section was generated from the binding values

Fig. 2 The diagnostic values of LSM and combination of LSM, FIB-4 and APRI for liver fibrosis. AUCS of LSM for the diagnosis of liver fibrosis was 0.740 (95\% Cl: 0.543-0.938); Of FIB-4 combined LSM was 0.758 (95\% Cl: 0.555-0.961); Of APRI combined LSM was 0.761 (95\% Cl: 0.580-0.942); Of combination of LSM, APRI and FIB-4 was 0.771 (95\% Cl: 0.580-0.942)

FIB-4 (0.509),the differences of which reached statistical significance ( $\mathrm{Z}$ were equal to 3.636 respectively while $P$ were equal to 0.000$)$. The $A U C$ value (0.869) of significant liver fibrosis by the combination of LSM value and APRI value as well as FIB-4 index was higher than that of LSM value (0.849). There were no significant statistical differences in this case when $Z$ was equal to 0.208 and $P$ was equal to 0.418. The values of $S e, S p, P P V, N P V,+L R$, and $-L R$ in the diagnosis of significant liver fibrosis with biochemistry indicators were listed. They were clearly shown in the following Table 3, Figs. 4, and 5.

\section{Discussion}

TE is a relatively mature non-invasive diagnostic technique for liver fibrosis. The ultrasonic liver cirrhosis detector used in our study is an operation instrument based on the principle of TE. It has the same advantages of noninvasiveness and good repetitiveness as Fibroscan and Fibrotouch. This study showed that TE and APRI were effective in diagnosing young $\mathrm{CHB}$ patients with liver fibrosis, with an $A U C$ value above 0.700 . TE and APRI could perform well in differentiating the stages of liver fibrosis in children with CHB. The results of LSM and APRI in the diagnosis of CHB patients with significant liver fibrosis were good, with the $A U C$ values all being more than 0.700 . It was shown that $\mathrm{TE}$ can better differentiate patients with significant liver fibrosis than APRI. The diagnostic value of TE was better than that of APRI and FIB-4 for CHB children with significant liver fibrosis. The combination of TE and multi-parameter indicators failed to significantly improve their abilities in the diagnosis of varying degrees of liver fibrosis in our study.

Our results showed that TE was superior to APRI and FIB-4 index in the diagnosis of hepatic fibrosis in terms of value, which was consistent with the results obtained by Orasan et al. [10] and Guo Feng et al. [11]. Teshale and colleagues found that the FIB-4 index can better differentiate mild liver fibrosis from significant liver fibrosis in adults with CHB [12]. However, results from our research suggested that the FIB-4 index failed to differentiate mild liver fibrosis from significant liver fibrosis in children with CHB. A study by Wang et al. showed that age has a bearing on the accuracy of the 


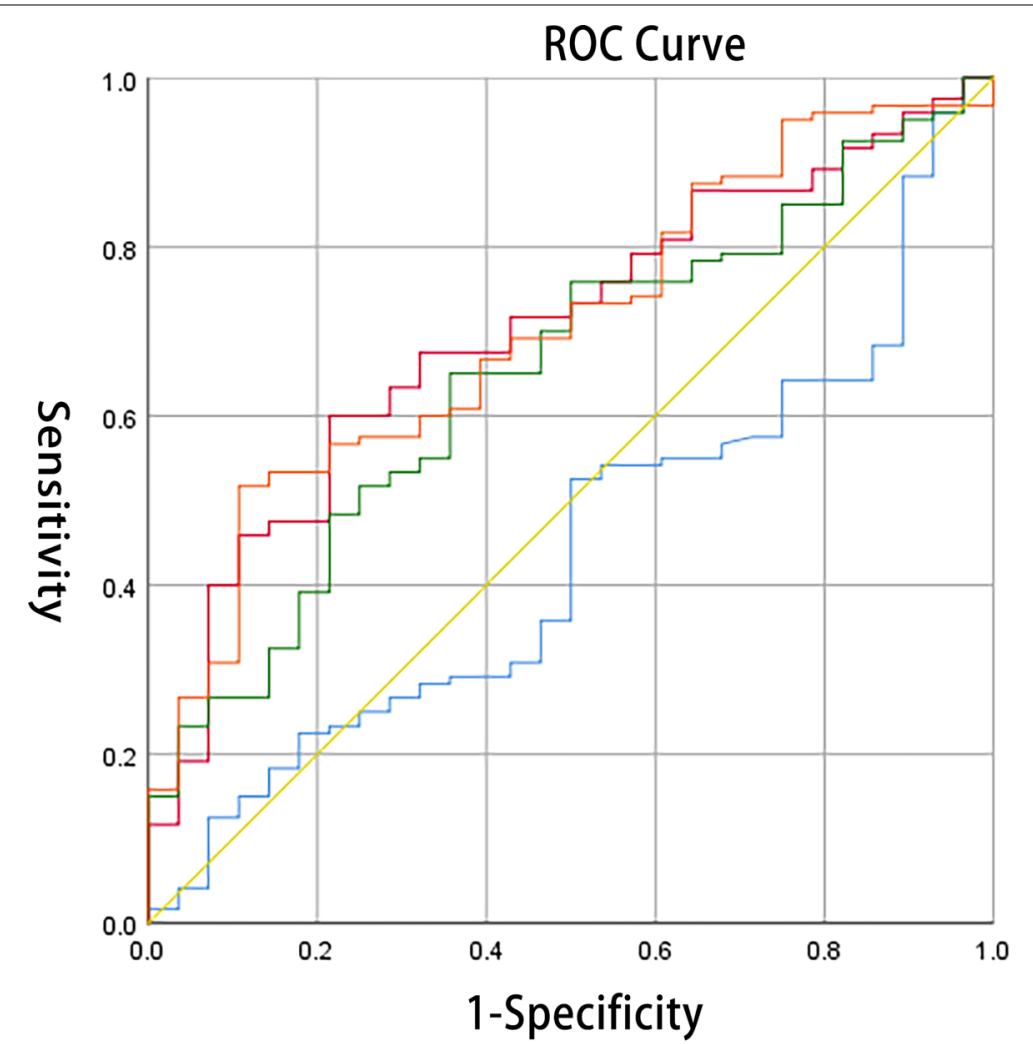

\section{Source of Curves}

AAR

- APRI

-FIB-41

- APRI+FIB-4

- Reference line

Diagonal section was generated from the binding values

Fig. 3 The diagnostic values of APRI, FIB-4 and AAR for liver fibrosis. AUCS of APRI for the diagnosis of liver fibrosis was 0.701 (95\% Cl: 0.603-0.800); Of FIB-4 was 0.651 (95\% Cl: 0.546-0.755); Of AAR was 0.440 (95\% Cl: 0.328-0.552); Of the combination of APRI and FIB-4 was 0.703 (95\% Cl: $0.605-0.801)$

Table 3 The area under ROC curve and its relevant parameters of significant liver fibrosis diagnosed by four non-invasive diagnostic indicators single or in combination

\begin{tabular}{|c|c|c|c|c|c|c|c|c|c|c|c|c|c|}
\hline Parameters & $\mathrm{n}$ & $A \cup C$ & $P$ & $95 \% \mathrm{Cl}$ & Cut-off & $\mathrm{Se}(\%)$ & $\mathrm{Sp}(\%)$ & PPV (\%) & NPV (\%) & $+\mathrm{LR}$ & $-\mathrm{LR}$ & $P$ & $x^{2}$ \\
\hline APRI & 148 & 0.701 & 0.001 & $0.591-0.810$ & 0.76 & 61.54 & 72.95 & 32.70 & 89.90 & 2.28 & 0.53 & & \\
\hline AAR & 148 & 0.458 & 0.500 & $0.329-0.586$ & 1.13 & 69.23 & 48.36 & 22.20 & 88.10 & 1.45 & 0.76 & & \\
\hline FIB-4 & 148 & $0.509(2)$ & 0.884 & $0.388-0.630$ & 0.08 & 84.62 & 27.87 & 20.00 & 89.50 & 1.17 & 0.55 & & \\
\hline$A P R I+F \mid B-4$ & 148 & 0.684 & 0.003 & $0.565-0.802$ & 0.16 & 61.54 & 70.49 & 30.80 & 89.60 & 2.09 & 0.55 & 0.970 & 6.698 \\
\hline LSM & 43 & 0.849 (1) & 0.001 & $0.713-0.986$ & $8.4 \mathrm{kPa}$ & 81.82 & 78.12 & 56.20 & 92.60 & 3.74 & 0.23 & & \\
\hline$A P R I+L S M$ & 43 & 0.866 & 0.000 & $0.740-0.993$ & 0.15 & 90.91 & 71.87 & 52.60 & 95.80 & 3.23 & 0.13 & 0.634 & 6.122 \\
\hline FIB-4 + LSM & 43 & 0.855 & 0.001 & $0.723-0.986$ & 0.24 & 81.82 & 78.12 & 56.20 & 92.60 & 3.74 & 0.23 & 0.570 & 6.698 \\
\hline $\mathrm{APRI}+\mathrm{FIB}-4+\mathrm{LSM}$ & 43 & 0.869 & 0.000 & $0.741-0.998$ & 0.47 & 63.64 & 96.87 & 87.50 & 88.60 & 20.36 & 0.38 & 0.797 & 4.619 \\
\hline
\end{tabular}

(1)Compared with APRI, $Z=1.650, P=0.049$; (1)compared with FIB-4, $Z=3.636, P=0.000$; (1)compared with APRI + FIB-4 + LSM, $Z=0.208, P=0.418$

FIB-4 index in the diagnosis of significant liver fibrosis [13]. As thus, there is a need to take the effect of age into account in the application of the FIB-4 index. The AAR values failed to perform well in the diagnosis of CHB patients with varying degrees of liver fibrosis, with $A U C$ values all being less than 0.700 , which was in accord with the findings of Eminler et al. [14]. However, there is still space for improvement with regard to the considerations of the test results influenced by the sample size and the cooperative degree of the children in the process of the tests and other factors. There is also a need for us to verify the results with a larger sample size so as to reduce the influence of external factors. 


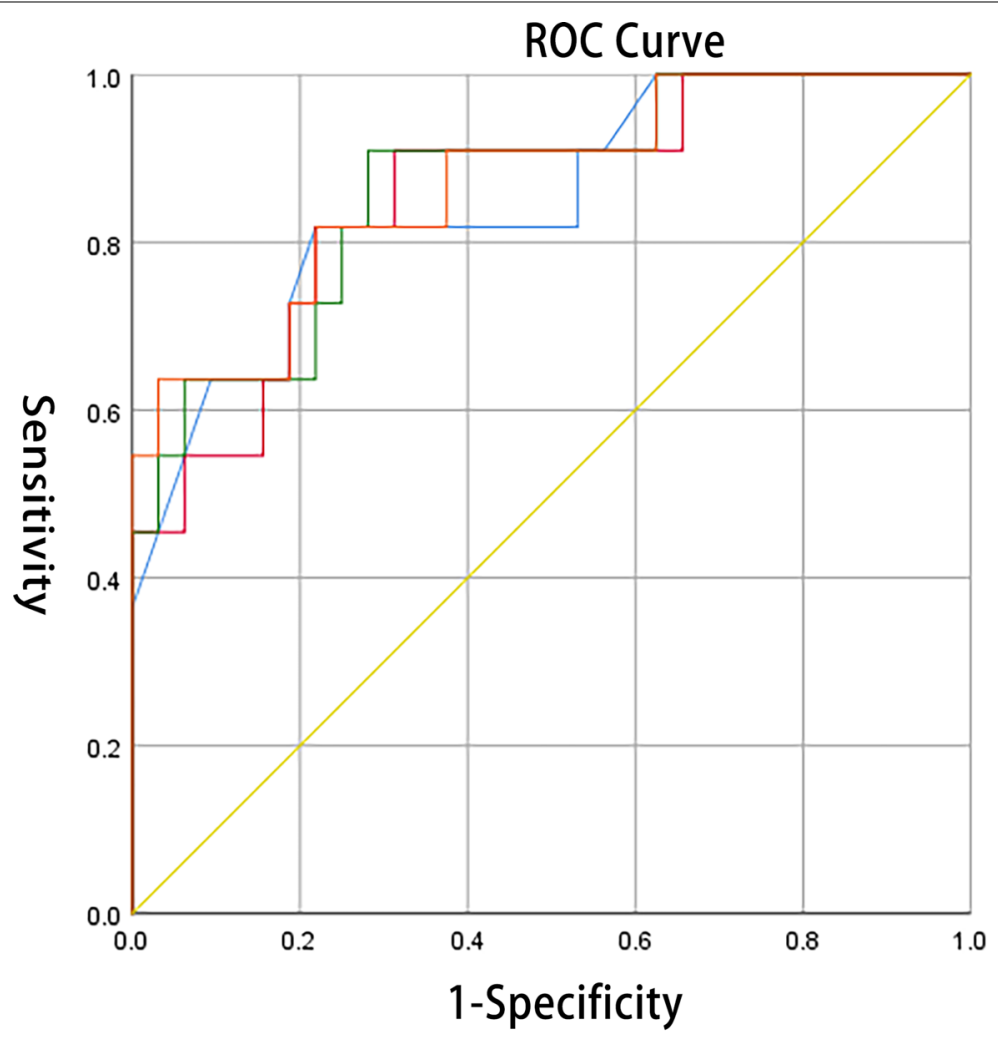

\section{Source of Curves}

- LSM

- FIB-4+LSM

APRI+LSM

- APRI+FIB-4+LSM

Reference line

Diagonal section was generated from the binding values

Fig. 4 The diagnostic values of LSM and a combination of LSM, FIB-4 and APRI for significant liver fibrosis. AUCS of LSM for the diagnosis of significant liver fibrosis was 0.849 (95\% Cl: 0.713- 0.986); Of FIB-4 combined LSM was 0.855 (95\% Cl: 0.723-0.986); Of APRI combined LSM was 0.866 (95\% Cl: 0.740-0.993); Of combination of LSM, APRI and FIB-4 was 0.869 (95\% Cl: 0.741-0.998)

The $A U C$ values were calculated to analyze the diagnostic value of TE and its combination of different parameters on liver fibrosis. The results showed that the combination of TE and multi-parameter indicators failed to significantly improve their abilities in the diagnosis of varying degrees of liver fibrosis, which was complied with the results by Zeng et al. [15]. However, it was also found that the combination of APRI and FIB-4 index can improve the diagnostic capacity for liver fibrosis of patients with CHB [16]. The sample size, age and other factors of the study should be taken into account.

Our results showed that the best cut-off values of LSM for the diagnosis of liver fibrosis and significant liver fibrosis were $5.9 \mathrm{kPa}$ and $8.4 \mathrm{kPa}$, respectively. In this case, the sensitivities were $94.12 \%$ and $81.82 \%$, and specificities were $55.56 \%$ and $78.12 \%$, respectively. Goyal R. found that adults with liver fibrosis in stage F2, F3 can be excluded by FibroScan measured LSM value $<6 \mathrm{kPa}$, and LSM value $>9 \mathrm{kPa}$ indicated that the degree of liver fibrosis reaches stage F2 or F3, and its sensitivity and specificity were above $90 \%$ [17]. Chang
P. E. and others found that the optimal cut-off for LSM diagnostic significant liver fibrosis $(F \geq 2)$ in adults was $9.0 \mathrm{kPa}$, whose sensitivity and specificity were $67.4 \%$ and $75.4 \%$, respectively [18]. And a recent study found that the cut-off values (specificity,sensitivity) for significant fibrosis and advanced fibrosis were $5.6 \mathrm{kPa}$ (75.7\%, 67.4\%), $6.9 \mathrm{kPa}(91.5 \%, 81.3 \%)$ respectively in CHB children aged 0-6 years [6]. The research of $\mathrm{Xu} \mathrm{ZQ}$ and colleagues showed that the value of LSM increased with age, as the cut-off values for significant liver fibrosis $(F \geq 2)$ and progressive liver fibrosis $(F \geq 3)$ in children under 12 years old were 5.8 and $7.0 \mathrm{kPa}$, respectively, and they were 6.6 and $8.0 \mathrm{kPa}$ for children above 12 years old [7]. The best cut-off values of LSM in these researches were somewhat different. As for the factors influencing the LSM values, Lin et al. [19] found that the LSM values of CHB patients may be in connection with the level of AST, ALT, total bilirubin, and albumin. The studies conducted by Piscaglia F. and other scholars [20] found that most detectors with TE as the principle were in intermediate consistency with 


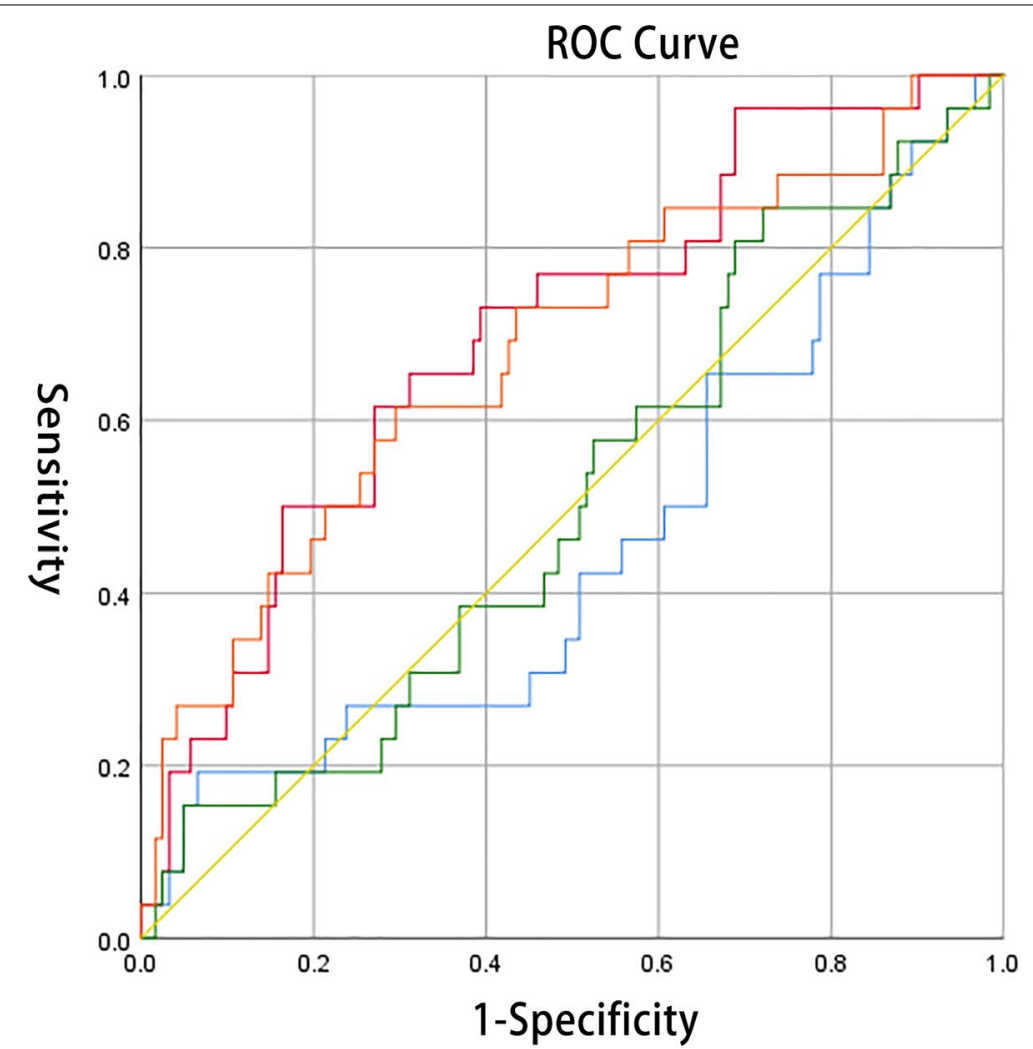

\section{Source of Curves}

- AAR

- APRI

-FIB-41

- APRI+FIB-4

Reference line

\section{1-Specificity}

Fig. 5 The diagnostic values of APRI, FIB-4 and AAR for significant liver fibrosis. AUCS of APRI for the diagnosis of significant liver fibrosis was 0.701 (95\% Cl: $0.591-0.810) ;$ Of FIB-4 was 0.509 (95\% Cl: 0.388-0.630); Of AAR was 0.458 (95\% Cl: 0.329-0.586); Of the combination of APRI and FIB-4 was 0.684 (95\% Cl: 0.565-0.802)

the results of Fibroscan, indicating that the general use of Fibroscan thresholds for defining the stage of liver fibrosis in all new machines was not feasible. So, the effects of operating instruments, ALT, AST, age of the patients and other clinical parameters, as well as the cooperative degree of children in the process of operation on TE should be considered. The influence factors of TE technology need to be studied by more data. The sample size needs to be further enlarged to explore the cut-off value for defining the stages of $\mathrm{CHB}$ patients with liver fibrosis by TE.

This study had several limitations. First, the number of patients, especially the sample size of patients with the F3-F4 fibrosis stage was small, and the number of patients we selected who were performed TE was small. Second, in the practical operation of TE testing, the difficulty to cooperate with young children and the impact of related factors such as the age, BMI and laboratory indicators of the children could interfere with the accuracy.Third, there are five scoring systems to evaluate the degree of liver fibrosis. In this study, we used Scheuer scoring systems, which might not be the most accurate one. Further studies to compare the accuracy of the different scoring systems are necessary. Future studies need to utilize larger sample sizes and stratify the patients according to their age, BMI and laboratory indicators.

\section{Conclusion}

Transient elastography performed better than APRI and FIB-4 index in the diagnosis of young CHB patients with significant liver fibrosis, with a relatively high application value for the clinical diagnosis of $\mathrm{CHB}$ patients with varying degrees of liver fibrosis. However, we need to consider the impact of relevant clinical factors on the test results in the process of application.

\section{Abbreviations}

ALT: Alanine aminotransferase; AST: Aspartate aminotransferase; AUC: The area under receiver operating characteristic; APRI: Aspartate aminotransferase (AST) -to- platelet (PLT) ratio index; AAR: AST-to-ALT ratio; CHB: Chronic hepatitis B; HBV: Hepatitis B virus; LB: Liver biopsy; LSM: Liver stiffness measurement; $-L R$ : Negative likelihood ratio; $+\angle R$ : Positive likelihood ratio; NPV: Negative predictive value; PLT: Platelet; PPV: Positive predictive value; ROC: Receiver operating characteristic; Se: Sensitivity; Sp: Specificity.

\section{Acknowledgements}

The authors are grateful to all patients who volunteered to participate in the study, and we also appreciate the support of the data collectors. This study 
was supported by a grant from the Hunan Provincial Development and Reform Commission in China (Grant [2019]875). The funding institution had no role in study design, data collection and analysis, decision to publish, or preparation of the manuscript. No additional external funding was given to the study.

\section{Authors' contributions}

LHY, LSJ and QJ conceived and designed the study. LHY, LSJ, QJ, PSX, OYWX, TYF, JT and TL performed the experiments. LHY, LSJ, QJ, TYF, JT and ZCC analyzed the data. LSJ, QJ, TL, PSX and OYWX contributed reagents/materials/ analysis tools. QJ wrote the manuscript. All authors read and approved the final manuscript.

\section{Funding}

This study was supported by a Grant from the Hunan Provincial Development and Reform Commission in China (Grant [2019] 875).

\section{Availability of data and materials}

The data and materials used and analyzed in this study are available from corresponding author on reasonable request.

\section{Declarations}

\section{Ethics approval and consent to participate}

This study was approved by the Ethics Committee of Hunan Children's Hospital (IRB No. HCHLL-2019004), and obtained the formal written informed consent from all the parents and guardians of the participating children. All data collection from participants was fully anonymous. All experiments were performed in accordance with the approved guidelines and regulations.

\section{Consent for publication}

Not applicable.

\section{Competing interests}

The authors declare that they have no competing interests.

\section{Author details}

'Department of Hepatology, Hunan Children's Hospital, Changsha 410007. China. ${ }^{2}$ Department of Maternal and Child Health, Xiangya School of Public Health, Central South University, Changsha 410078, China. ${ }^{3}$ Pediatrics Research Institute of Hunan Province, Hunan Children's Hospital, Changsha 410007, China. ${ }^{4}$ Academy of Pediatrics of University of South China, Hengyang 421001, China.

Received: 15 October 2021 Accepted: 8 February 2022

Published online: 18 February 2022

\section{References}

1. Chinese Society of Hepatology, Chinese Society of Infectious Diseases. Prevention and treatment for chronic hepatitis B: interpretation of Chinese guideline (2015 version). J Clin Hepatobiliary Dis. 2015;31: 1941-1960.

2. Ismail MH, Pinzani M. Reversal of liver fibrosis. Saudi I Gastroenterol. 2009;15:72-9. https://doi.org/10.4103/1319-3767.45072.

3. Chang CY, Martin P, Fotiadu A, Hytiroglou P. A patient with chronic hepatitis $B$ and regression of fibrosis during treatment. Semin Liver Dis. 2010;30:296-301. https://doi.org/10.1055/s-0030-1262515.

4. Kose S, Ersan G, Tatar B, Adar P, Sengel BE. Evaluation of percutaneous liver biopsy complications in patients with chronic viral hepatitis. Eurasian J Med. 2015;47:161-4. https://doi.org/10.5152/eurasianjmed.

5. Shijie J, Xinghua T. Application of FbroScan combined with APRI and HA for liver fibrosis in patients with chronic hepatitis B. J Hepatobiliary Surg 2017;25:285-8.

6. Xu Z, Zhao J, Liu J, Dong Y, Wang F, Yan J, et al. Assessment of liver fibrosis by transient elastography in young children with chronic hepatitis B virus infection. Hepatol Int. 2021;15:602-10. https://doi.org/10.1007/ s12072-021-10194-7.
7. ZhiQiang Xu, Yi D, FuChuan W, LiMin W, JianGuo Y, LiLi C, Wang Pu, AiQin $\mathrm{Li}$, YanWei Z, Min Z. Value of transient elastography in the diagnosis of liver fibrosis in chronic hepatitis B children of different ages. J Clin Hepatol. 2020;36:1268-72.

8. Teufel-Schäfer U, Flechtenmacher C, Fichtner A, Hoffmann GF, Schenk JP, Engelmann G. Transient elastography correlated to four different histological fibrosis scores in children with liver disease. Eur J Pediatr. 2021;180:2237-44. https://doi.org/10.1007/s00431-021-04001-6.

9. Qiu J, Yan H, Cheng N, Lu X, Hu X, Liang L, Xiao Z, Tan L. The clinical and epidemiological study of children with hand, foot, and mouth disease in Hunan, China from 2013 to 2017. Sci Rep. 2019. https://doi.org/10.1038/ s41598-019-48259-1.

10. Orasan OH, lancu M, Sava M, Saplontai-Pop A, Cozma A, Sarlea ST, et al. Non-invasive assessment of liver fibrosis in chronic viral hepatitis. Eur J Clin Invest. 2015;45:1243-51. https://doi.org/10.1111/eci.12543.

11. Guo F, Zhuang XF, Wang XB, et al. Evaluation of three non-invasive diagnosis of liver fibrosis for the diagnosis of chronic hepatitis B progressive liver fibrosis (>F3). Chin Hepatol. 2018;23:235-8.

12. Teshale E, Lu M, Rupp LB, Holmberg SD, Moorman AC, Spradling P, et al. APRI and FIB-4 are good predictors of the stage of liver fibrosis in chronic hepatitis B: the Chronic Hepatitis Cohort Study (CHeCS). J Viral Hepat. 2014;21:917-20. https://doi.org/10.1111/jvh.12279.

13. Wang W, Zhao X, Li G, Wang L, Chen Y, Ma K, et al. Diagnostic thresholds and performance of noninvasive fibrosis scores are limited by age in patients with chronic hepatitis B. J Med Virol. 2019;91:1279-87. https:// doi.org/10.1002/jmv.25435.

14. Eminler AT, AyyildizT, Irak K, Kiyici M, Gurel S, Dolar E, et al. AST/ALT ratio is not useful in predicting the degree of fibrosis in chronic viral hepatitis patients. Eur J Gastroenterol Hepatol. 2015;27:1361-6. https://doi.org/10. 1097/MEG.0000000000000468.

15. Zeng X, Xu C, Li M, Xia J, Liu M, Zhu P, et al. The diagnostic value of FibroScan in assessing significant liver fibrosis in patients with chronic hepatitis B. West Indian Med J. 2015;65:106-10. https://doi.org/10.7727/wimj.2014. 150.

16. Ben Ayed H, Koubaa M, Yaich S, Rekik K, Ben Jemaa T, Maaloul I, et al. A new combined predicting model using a non-invasive score for the assessment of liver fibrosis in patients presenting with chronic hepatitis $B$ virus infection. Med Mal Infect. 2019;49:607-15. https://doi.org/10.1016/j. medmal.2019.02.004.

17. Goyal R, Mallick SR, Mahanta M, Kedia S, Shalimar, Dhingra R, et al. Fibroscan can avoid liver biopsy in Indian patients with chronic hepatitis B. J Gastroenterol Hepatol. 2013;28:1738-45. https://doi.org/10.1111/jgh. 12318.

18. Chang PE, Hartono JL, Ngai YL, Dan YY, Lim KB, Chow WC. Optimal liver stiffness measurement values for the diagnosis of significant fibrosis and cirrhosis in chronic liver disease in Singapore. Singapore Med J. 2019;60:532-7. https://doi.org/10.11622/smedj.2018156.

19. Lin Z, Liang J, Zhu J, Hu C, Gu Y, Lai J, et al. Diverse correlations between fibrosis-related factors and liver stiffness measurement by transient elastography in chronic hepatitis B. Eur J Gastroenterol Hepatol. 2018;30:21725. https://doi.org/10.1097/MEG.0000000000001023.

20. Piscaglia F, Salvatore V, Mulazzani L, Cantisani V, Colecchia A, Di Donato $\mathrm{R}$, et al. Differences in liver stiffness values obtained with new ultrasound elastography machines and Fibroscan: a comparative study. Dig Liver Dis. 2017:49:802-8. https://doi.org/10.1016/j.dld.2017.03.001.

\section{Publisher's Note}

Springer Nature remains neutral with regard to jurisdictional claims in published maps and institutional affiliations. 